\title{
ON CONVEX IDEALS
}

\section{CARL W. KOHLS}

In a partially ordered ring, the convex ideals are of special interest because their quotient rings possess a naturally induced order [3, 5.2]. Some partially ordered rings have an abundance of convex ideals; for instance, every prime ideal in the ring of all continuous real-valued functions on a topological space is convex (with respect to the usual order) $[3,5.5]$. The results presented here concern those orders on a ring with respect to which every primitive ideal is convex, or every prime ideal is convex. In particular, we give an example of a commutative partially ordered ring with identity in which every maximal ideal is convex but not every prime ideal is convex.

A partial order on a ring $A$ can be defined by specifying a set $P \subset A$ called the positive cone, satisfying the conditions: $P+P \subset P, P P \subset P$, and $P \cap-P=\{0\}$. For convenience, we shall identify a partial order on a ring with the positive cone that defines it. An ideal $I$ in $A$ is $P$-convex if $a \in P, b-a \in P$, and $b \in I$ imply $a \in I$.

The ring of all continuous real-valued functions on a topological space $X$ is denoted by $C(X)$. We use the symbol $\mathrm{R}$ for the field of real numbers, and $Q$ for the field of rational numbers. The one-point compactification of the countable discrete space $\mathrm{N}$ is denoted by $\mathrm{N}^{*}$; the point at infinity is designated by $\omega$. The same letter will be used for an ideal $I$ in $A$ and the natural homomorphism of $A$ onto $A / I$; thus, the image of $a \in A$ in $A / I$ is denoted by $I(a)$.

Observe that $\{0\}$ is a positive cone, and that every ideal in any ring is trivially $\{0\}$-convex. It is evident that the union of a chain of positive cones of a ring is again a positive cone, so that Zorn's Lemma implies the existence of a maximal order on any ring. The following statement is also easily verified.

For any family $\mathcal{F}$ of ideals in a ring $A$, let $\mathcal{C}$ be the collection of orders $P$ on $A$ such that every ideal in $\mathfrak{F}$ is $P$-convex. Then $\mathcal{C}$ is nonempty, and every order in $\mathbb{C}$ is contained in a maximal order in $\mathbb{C}$.

In particular, there is a maximal order on $A$ with respect to which every primitive ideal in $A$ is convex, and a maximal order on $A$ with respect to which every prime ideal in $A$ is convex.

From now on, we consider mainly commutative semisimple rings.

Definition. Let $A$ be a commutative semisimple ring, and let $\left\{M_{\gamma}\right\}$ be the family of primitive (i.e., prime maximal) ideals in $A$.

Received by the editors December 24, 1965. 
The pointwise order on $A$ corresponding to a family of orders on the quotient fields $A / M_{\gamma}$ is the order $\left\{a \in A: M_{\gamma}(a) \geqq 0\right.$ in $A / M_{\gamma}$ for each $M_{\gamma}$. .

It is straightforward to verify that this actually gives an order; it is called the natural order in [2]. As already indicated, every field can be ordered. The so-called "unorderable" fields are those possessing no total order.

Following Fine, Gillman, and Lambek $[1,8.2]$, we say that an order $P$ on a commutative ring $A$ is quasi-real if $a^{2} \in P$ for all $a \in A$. Clearly, a pointwise order on a semisimple ring $A$ is quasi-real if and only if it corresponds to a family of quasi-real orders on the quotient fields of $A$.

Let $\mathfrak{T}$ denote the collection of orders on $A$ with respect to which every primitive ideal in $A$ is convex. Obviously, if $P \in \mathscr{M}$ and $P^{\prime} \subset P$, then $P^{\prime} \in \mathscr{M}$.

ThEOREM. Let $A$ be a commutative semisimple ring. Every pointwise order on $A$ is in $\mathfrak{M}$; and every order in $\mathfrak{T C}$ is contained in a pointwise order. Every maximal order in TT is a pointwise order that corresponds to a family of maximal orders; and a pointwise order that induces a family of maximal orders is maximal in $\mathfrak{T}$. Every quasi-real maximal order in $\mathfrak{N}$ is a pointwise order that corresponds to a family of total orders; and a pointwise order that induces a family of total orders is quasi-real and maximal in $\mathfrak{T}$.

Proof. Let $P_{0}$ be any pointwise order on $A$. For any primitive ideal $M$ in $A$, if $a \in P_{0}$ and $b-a \in P_{0}$, then $0 \leqq M(a) \leqq M(b)$ in the given order on $A / M$. Thus, $b \in M$ implies $a \in M$, whence $M$ is $P_{0^{-}}$ convex. Therefore $P_{0} \in \mathfrak{T}$.

Now any $P_{1} \in \Re($ induces an order on every quotient field of $A$. Let $P_{2}$ be the pointwise order on $A$ corresponding to the resulting family of orders on the quotient fields. For each primitive ideal $M$ in $A, a \in P_{1}$ implies $M(a) \geqq 0$ in the induced order on $A / M$; thus, $a \in P_{2}$. It follows that $P_{1} \subset P_{2}$. (But $P_{2}$ need not coincide with $P_{1}$; see Remark 5 below.)

It has been shown that every order in $\mathscr{T}$ is contained in a pointwise order in $\mathbb{T}$; hence any maximal order in $\mathfrak{T}$ is a pointwise order. Each order in the family to which a maximal order $P^{*}$ in $\mathfrak{T}$ corresponds can be extended to a maximal order. Then the pointwise order in $\mathscr{T}$ corresponding to the resulting family contains $P^{*}$, and hence coincides with $P^{*}$, by maximality. Thus, $P^{*}$ corresponds to a family of maximal orders.

Next, assume that $P$ is a pointwise order that induces a family of maximal orders, and let $P^{\prime}$ be a maximal order in $\mathfrak{N}$ containing $P$. Consider any primitive ideal $M$ in $A$. Denote the orders on $A / M$ 
that $P$ and $P^{\prime}$ induce by $I$ and $I^{\prime}$, respectively, and denote the orders on $A / M$ in the families to which $P$ and $P^{\prime}$ correspond by $Q$ and $Q^{\prime}$, respectively. If $M(a) \in I$, then there exists $b \in P$ such that $M(b)$ $=M(a)$; and $M(b) \in Q$ by definition of $P$. It follows that $I \subset Q$. Similarly, $I^{\prime} \subset Q^{\prime}$. Also if $M(a) \in I$, then for $b \in P$ such that $M(b)=M(a)$ we have $b \in P^{\prime}$, whence $M(b) \in I^{\prime}$. Thus, $I \subset I^{\prime}$. Since $I$ is maximal by assumption, we have $I=Q$ and $I=Q^{\prime}$, so that $Q=Q^{\prime}$. As this holds for all primitive ideals in $A$, we have $P=P^{\prime}$. Therefore $P$ is maximal in $\mathfrak{T}$. (In general, $I$ need not coincide with $Q$; see Remark 6 below.)

The last statement now follows easily from the result that any maximal quasi-real order on a field is total $[1,8.5]$, and the obvious fact that any total order is quasi-real and maximal.

CoRollary. The usual order on a ring of continuous functions $C(X)$ is a maximal order with respect to which every maximal ideal is convex.

Proof. The orders induced on the quotient fields of a ring of continuous functions $C(X)$ by the usual order are total $[3 ; 5.5]$; and the usual order is a pointwise order, since a function is nonnegative at each point of $X$ if and only if its image in every quotient field is nonnegative.

REMARKs. (1) It follows immediately from the Corollary and $[3,5.5]$ that the usual order on $C(X)$ is a maximal order with respect to which every prime ideal is convex.

(2) A pointwise order need not be maximal in $\mathscr{T}$. Any order on a field is a pointwise order; and some fields have orders that are not maximal.

(3) In contrast to $[1,8.5]$, if a maximal order on a field is not quasi-real, it need not be total. Indeed, any field has a maximal order; and some fields have no total order.

(4) Obviously, if $A$ is a commutative semisimple ring such that no quotient field has a nontrivial order, then $\{0\}$ is the only order in $\mathfrak{T}$. The ring of integers is such a ring.

(5) The pointwise order corresponding to a family of orders induced on the quotient fields of $A$ by an order in $\mathfrak{T}$ need not coincide with it. For example, let $A$ be the direct sum of two copies of $\mathrm{R}$, and let $P_{1}=\{(r, s): r \geqq s \geqq 0\}[3,5 \mathrm{~B} .4]$. Then the order $P_{2}$ corresponding to the family of orders induced on the quotient fields by $P_{1}$ is $P_{2}=\{(r, s): r \geqq 0, s \geqq 0\}$. Note also that $P_{1}$ is an order in $\mathfrak{N}$ that is not a pointwise order, since the order on a quotient field induced by a pointwise order is contained in the order belonging to the family to which the pointwise order corresponds. We observe that both $P_{1}$ and $P_{2}$ make $A$ a lattice-ordered ring, providing a counterexample to 
$[2,4.1]$, which was known to be erroneous (Math. Reviews 19 (1958), 1156).

(6) The family of orders induced on the quotient fields by a pointwise order need not coincide with the family of orders to which it corresponds, even if every order in the latter family is total. For example, let $\theta$ be the positive square root of 2 (in the usual order on $\mathrm{R})$, and let $A$ be the subring of functions in $C\left(\mathrm{~N}^{*}\right)$ with values in $Q(\theta)$. We denote the maximal ideal $\{a \in A: a(\omega)=0\}$ by $M_{\omega}$. Each quotient field of $A$ is isomorphic to $Q(\theta)$. We totally order all quotient fields except $A / M_{\omega}$ by specifying that $Q$ have the usual order and $\theta$ be positive; we order $A / M_{\omega}$ similarly but with $-\theta$ positive. If $P$ is the pointwise order on $A$ corresponding to this family of orders, then no element of $A$ whose image in $A / M_{\omega}$ is $-\theta$ can be in $P$. Thus, $-\theta$ is not positive in the induced order on $A / M_{\omega}$.

(7) It follows easily from the Theorem that if each quotient field of $A$ has a unique maximal order, then there is a unique maximal order in $\mathfrak{T}$. (An example of a field that has a unique maximal partial order is $Q$; for further details, see [5].) The converse is not true, however. For example, let $A$ be the ring of all functions in $C\left(\mathrm{~N}^{*}\right)$ that have rational values at the points of $\mathrm{N}$. We denote the maximal ideal $\{a \in A: a(\omega)=0\}$ by $J_{\omega}$. Then the order $P$ on $A$ induced by the usual order on $C\left(\mathrm{~N}^{*}\right)$ is the unique maximal order in $\mathfrak{T}$. For, any maximal order in $\mathscr{N}$ is a pointwise order corresponding to the same family as that to which $P$ corresponds, except perhaps on $A / J_{\omega}$. But it is easy to see that this implies that the two orders coincide. On the other hand, $A / J_{\omega}$ is isomorphic to $\mathrm{R}$, which does not have a unique maximal partial order; for example, there is a maximal partial order on $\mathrm{R}$ in which $\pi$ is infinitely small relative to $Q$.

We now generalize the result on prime ideals mentioned in the introduction.

Propositron. Let $I$ be an ideal in a ring $A$, where $A=C(X)$ for some topological space $X$ (with the usual order), or $A$ is a commutative regular ring (with a pointwise order). Then every prime ideal in $I$ is convex.

Proof. As already mentioned, every prime ideal in $C(X)$ is convex with respect to the usual order. Now suppose $A$ is a commutative regular ring with a pointwise order $P$. Let $J$ be any ideal in $A$, and assume that $a \in P, b-a \in P$, and $b \in J$. Since $A$ is regular, there exist idempotents $e$ and $f$ in $A$ that generate the same ideals as $a$ and $b$, respectively. For any primitive ideal $M$ in $A, 0 \leqq M(a) \leqq M(b)$ in $A / M$. Now $M(e)$ and $M(f)$ can only be 0 or 1 . It follows that $M(e)=0$ if $M(f)=0$ and $M(f)=1$ if $M(e)=1$. Thus $M(e)=M(e) M(f)$ for each 
primitive ideal $M$. Since $A$ is semisimple, we have $e=e f$; this implies that $a \in J$. Therefore $J$ is $P$-convex.

By $[4,5.1]$, corresponding to each prime ideal $Q$ in $I$ there is a prime ideal $Q^{*}$ in $A$ such that $Q^{*} \cap I=Q$. It is easy to see that the convexity of $Q^{*}$ implies that of $Q$.

REMARKS. (8) The condition for convexity of prime ideals given in $[1,9.4]$ cannot be applied to ideals in $C(X)$; such ideals need not be $\pi$-rings (that is, squares need not have positive square roots). For example, the ideal generated by the identity function i in $C(\mathrm{R})$ is not a $\pi$-ring, since $\mathrm{i}^{2}$ has no positive square root in (i). The condition is also inapplicable in the case of a regular ring; an example is easily constructed from a ring having a quotient field with the trivial order.

(9) A prime ideal in a convex ideal need not be convex. For example, let $A$ be the direct sum of the ring of integers with $\mathrm{R}$, and let the order be $\{(n, r): n \geqq 0, r \geqq 0\}$. The ideal $I$ generated by $(1,0)$ in $A$ is convex; but the prime ideal generated by $(2,0)$ in $I$ is not convex.

The following example yields more information.

EXAMPLE. $A$ partially ordered commutative ring with identity such that every maximal ideal is convex but not every prime ideal is convex. Let $\mathrm{R}[x]$ be the ring of polynomials over $\mathrm{R}$ with the usual order, and define $A$ to be the ring of formal power series in $y$ over $\mathrm{R}[x]$ with constant terms in $\mathrm{R}$. Let the order on $A$ be $\left\{\sum_{i=0}^{\infty} p_{i} y^{i} \in A\right.$ : the first nonzero $p_{i}$ is positive $\}$. The unique maximal ideal in $A$ is $\left\{\sum_{i=0}^{\infty} p_{i} y^{i} \in A: p_{0}=0\right\} ;$ it is easy to see that it is convex. Denote the ideal $\left\{\sum_{i=1}^{\infty} p_{i} y^{i}: p_{i} \in\left(x^{2}+1\right)\right\}$ by $Q$. Since $0 \leqq y \leqq\left(x^{2}+1\right) y$, $\left(x^{2}+1\right) y \in Q$, but $y \notin Q$, the ideal $Q$ is not convex. To see that $Q$ is a prime ideal in $A$, assume that $\sum_{i=0}^{\infty} a_{i} y^{i}, \sum_{i=0}^{\infty} b_{i} y^{i} \in Q$. Let $a_{n}$ and $b_{m}$ denote the first coefficients that fail to be in $\left(x^{2}+1\right)$. Then, in the coefficient $\sum_{j=0}^{n+m} a_{j} b_{n+m-j}$ of the product, we have $a_{j} \in\left(x^{2}+1\right)$ for $j<n$ and $b_{n+m-j} \in\left(x^{2}+1\right)$ for $j>n$. Thus, each term except $a_{n} b_{m}$ belongs to $\left(x^{2}+1\right)$. It follows that $\sum_{j=0}^{n+m} a_{j} b_{n+m-j} \notin\left(x^{2}+1\right)$, whence $\left(\sum_{i=0}^{\infty} a_{i} y^{i}\right)\left(\sum_{i=0}^{\infty} b_{i} y^{i}\right) \notin Q$.

\section{REFERENCES}

1. N. J. Fine, L. Gillman and J. Lambek, Rings of quotients of rings of functions, McGill Univ. Press, Montreal, 1965.

2. L. Gillman, Rings with Hausdorff structure space, Fund. Math. 45 (1957), 1-16.

3. L. Gillman and M. Jerison, Rings of continuous functions, Van Nostrand, Princeton, N. J., 1960.

4. C. W. Kohls, The space of prime ideals of a ring, Fund. Math. 45 (1957), 17-27.

5. C. W. Kohls and J. D. Reid, Orders on commutative rings, Duke Math. J. 33 (1966), 657-666. 